\title{
Predicting Results from Interaction Patterns during Online Group Work
}

\author{
Alvaro Figueira ${ }^{1}$ \\ ${ }^{1}$ CRACS \& INESC TEC - University of Porto, \\ Rua do Campo Alegre, 1021/1055, 4169-007 Porto, Portugal \\ arfedcc.fc.up.pt
}

\begin{abstract}
Group work is an essential activity during both graduate and undergraduate formation. Although there is a vast theoretical literature and numerous case studies about group work, we haven't yet seen much development concerning the assessment of individual group participants. The problem relies on the difficulty to have the perception of each student's contribution towards the whole work. We propose and describe a novel tool to manage and assess individual group. Using the collected interactions from the tool usage we create a model for predicting ill-conditioned interactions which generate alerts. We also describe a functionality to predict the final activity grading, based on the interaction patterns and on an automatic classification of these interactions.
\end{abstract}

Keywords: group work, individual assessment, interaction pattern, grading prediction, online tool.

\section{Introduction}

During the last decade, in many European countries the student / teacher ratio has continuously and systematically being increased. This situation is due to a more rational use of resources, as some would say; or due to measures to cut off public expenses, as another might also say. Apart from the reasons, there is a clear need for the use of other pedagogical resources and pedagogical means in order to promote a more decentralized education. In this article we present and describe a tool to manage, promote, predict behavior, and help to assess, learning activities developed in a group. Our view is that those activities should be increased because they are certainly a possible, simple solution, to circumvent this ratio problem.

The interaction between students, while undertaking a joint project, provides them an opportunity to have a more active role in their learning, and the possibility to develop their social skills. In fact, group work is a teaching strategy that makes students become more responsible for their learning and for the learning of their group fellows. Although the concept of "collaborative learning", also called "group work", is quite attractive in the first instance, it appears that it presents several difficulties for the teacher [1], especially at the time of evaluating and grading the students, as it is not always easy to have the perception of the contribution of each student to the whole 
group work. Accordingly, the adoption of this kind of pedagogical activity justifies the need for reliable instruments and techniques to provide a greater accuracy (and legitimacy) when assigning the student's final grading.

In the following sections we describe the problems and perspectives related with group work (section two); in section three we give a brief overview our tool. In section four we describe the interactions and the pattern mining to predict grading and, we conclude the article (section five) with a brief synthesis of the work.

\section{Group Work}

Modern pedagogy [1], [2] takes into account the need to expose students to collaborative work. However, group work is still far from being consensual and reluctance to its adoption derives from many handicaps of the respective assessment procedure.

One of the advantages of group work is that it provides students with an opportunity to improve their "social component" [3], which is essential given that we are in the "social networks generation". Other more objective aspect of the group work is the fact that every student has the opportunity to acquire new learnings, according to their knowledge / experiences and to the knowledge / experiences of the colleagues.

However, there are also disadvantages in conducting group work strategies. The main ones occur due to the large discrepancy with respect to the workload, or the engagement of each of the participants. To minimize these disadvantages some tools are used to analyse the development of the work, and to record which interactions are made [4].

\subsection{Assessment of Group Work}

Assessment is many times one of the most influential aspects when creating a new pedagogical activity. The way the work is evaluated and assessed largely depends on the purpose of it [5]. Taking into account the characteristics inherent in group work, it is essential to consider not just the final work but also the contribution of each element in the preparation of this work. We believe that there is a general consensus on respect to the need for differentiating the grades of participants in a group work. However, the same is not true when we discuss how this differentiation should be made. For example, three aspects have been proposed in [6]. Unfortunately, all of these characteristics are very hard to be objectively assessed in a short period of time.

However, there are many objective suggestions, for instance through auto and hetero evaluation [7]; through the creation of individual portfolios [8] and even through the use of basic level generic log-files, trace data, and "digests" to determine the interactivity degree in the course of the collaborative activity [9].

Knowing which strategy to adopt is still an open question, since every task has its own characteristics, namely on the modus operandi of group work, which makes it difficult to find a way that can be considered simultaneously advantageous to all the different cases. Despite the relevance of each of the aspects mentioned, it is important that the assessment should be as objective as possible. The problem is that some of 
aforementioned parameters are inherently subjective. Recently, we have seen an interesting proposal [10] which is based on relations between peers, and teachers.

\subsection{Group work in Online Environments}

The Moodle system is perhaps the most well-known and most-used worldwide, open source, learning management system. In Moodle there are activities which can be performed simultaneously by several students, whereas activities such as the Test, or the Survey, which typically require an individualized participation. It is curious to note that while it is possible to monitor the students' work in most Moodle activities, in none of them (except the Wiki) is possible to monitor the participation of each student individually. If we consider other LMSs which feature collaboration tools, we get essentially the same problems [2].

\section{Overview of the Philosophy of Our Proposed Tool}

We have proposed a tool to promote group work that is able to operate on two different modes, which correspond to different pedagogical situations. The first one, the "sequential activity" is based on the assumption that a group work is previously structured in tasks, one task per student. The second one if based on the view that students should structure themselves the work and organize them to be productive and accomplish the activity without further guidance. The tool works as a Moodle module and its management and usage characteristics have been described in [11].

\section{$4 \quad$ Pattern Mining From the Tool Usage}

While students use our tool in an online environment, provided by the Moodle LMS, their interactions are being stored in a new table, as log data, in the standard Moodle database. These interactions are atomic actions that a user performs in the context of the group tool usage.

We equipped the tool with the capability to distinguish, and identify up to eight different types of interactions (as listed in Table 1).

Table 1. List of the eight interactions.

\begin{tabular}{|cl|l|}
\hline 1. & Upload of a file & 5. Read a comment in a file \\
\hline 2. & Download of a file & 6. Grade a peer submission \\
\hline 3. & Edit/change a file & 7. Vote for accomplishment \\
\hline 4. & Register a comment in a file & 8. Grant permission for final submission \\
\hline
\end{tabular}

It is important to stress that a change to a file (interaction 3 ) can be done without doing a previous download (2), followed by a upload (1). In fact, a change to a file is an atomic operation which creates a new version of the same filename. On the other 
hand, a file upload is always the addition of a new file to the system, whereas, a file download means the access of a file without the immediate purpose of changing it.

\section{Examples of Interactions.}

Not only the interactions are important but also the order in which they appear. Sometimes there is a "logic" sequence of interactions. For example, it is expected that a student revises, or gets to know what a peer has done, before grading him/her. Nevertheless, illogic situations may arise from the interactions' sequence. For the sake of clarity, we present some examples:

- 5-5-3-4: the student read the comments of two different files, then decided to change one file and then updated a comment on the changed file.

- 2-2-6-6: the student downloaded two files and, without reading a single comment, graded two of his peers.

- 1-8: one student did an upload of a file, probably thought that he/she did his part of the work and granted permission for submission of the whole group work without looking at the peer's work.

\subsection{Data Analysis Moments}

Despite all user interactions with the tool being stored in the database immediately after being triggered, the system does not operate on "real-time". Actually, the automatic analysis of the interactions is triggered only on three moments, which seem to us of importance for pedagogical reasons: the first one occurs 48 hours after the first use of the tool by the students; the second one, occurs halfway of the time period defined for the whole group work, and; the last one occurs immediately after completion of the whole group job. We must say that the system is tuned for activities that last for at least one week.

The choice of these three stages is, respectively, due to:

First stage: was created due to the need to check if every student is motivated to perform the assigned job and understands what he/she is supposed to do;

Second stage: acts as a preliminary assessment stage, where early problematic situations may be detected providing the teacher with information for an eventual intervention/assistance;

Third stage: during the last stage all the data is collected, analysed, and stored in the Moodle database to be consulted during the next use of the tool.

\subsection{Data Mining and Analysis}

A stored database of previous interactions is provided to the system in order to have a starting point for the upcoming and future analysis and predictions.

There are two important analyses that must be considered: 1) the detection of an "illogical" pattern in a sequence, and; 2) the categorization of each sequence into a set of pre-defined categories for grade prediction. We illustrate the process of creating the 
clusters and of assigning a sequence of interactions to each cluster. To allow graphic representation of the dimensions we assume that each vector belongs to a 2dimensional space, say dimension V1 and dimension V2. This restriction of the illustration does not loose generality to the 8-dimensional model. On the left side of Fig. 1 (A) we depict three test-data vectors. Those vectors are manually picked as seeds for the creation of a particular cluster (either the "worst", "medium" or "best"). From these vectors, the system computes the centroid ( $\mathrm{P} 1)$ of the cluster. The process is repeated to create the three centroids (part B of the figure). Finally, in part C) of the figure we depict what happens when a vector (D1 or D2) is presented to the system: computing the distance to the nearest centroid the vector is assigned to a particular cluster. In Fig. 1, D1 was assigned to P1 and D2 to P3.

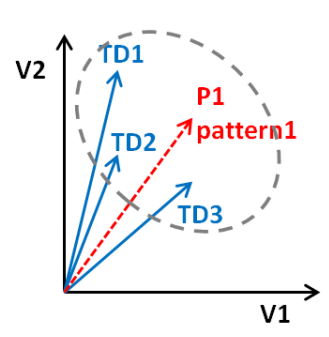

A)

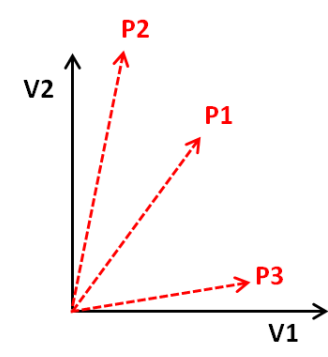

B)

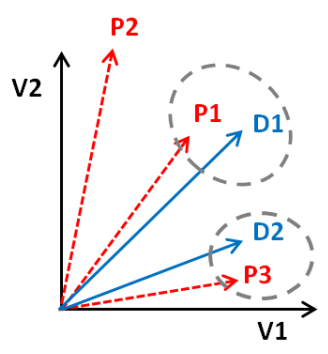

C)

Fig. 1. Clustering the interactions.

As a result of the processing phases A) and B) we get three groups: the group of students whose interaction patterns may lead to a higher grade, to a medium grade and to a lower grade. As a result of processing phase C) the system classifies each interaction in a cluster, therefore predicting the final grade. The system evolves by using the "medoids" (the vector that is the most close to geometric centre) of each resulting cluster, in each stage, and storing them in the Moodle database. These patterns are only made available for future system analysis when the final corresponding grade, given by the teacher, becomes available.

This is a self-feedback system that evolves as it is used. As the database increases and more patterns are acquired and by the system, its prediction accuracy gets improved.

\section{Conclusions}

Sometimes group assignments are a set of individual tasks, which later on are gathered and assessed as a single activity, where the participation of each group participant is seen as part of a "black box". More than frequently, the grading of each participant for the developed work is not meritocratic, but just the same as the mark given to the whole group work. 
Our system can help overcome the difficulties to evaluate the work and involvement of each group participant by allowing the teacher to verify the participation of each student. The tool allows the teacher to follow the progress of the group work and receive notification whenever a student creates a sequence of interactions with the tool that don't make sense or are clearly illogic. Moreover, at three specified stages, during the group work, the tool automatically predicts the final grade of each participant based on their interactions and on a database of previous interaction patterns.

\section{Acknowledgements}

This work is financed by ERDF - European Regional Development Fund through the COMPETE Programme (operational programme for competitiveness) and by National Funds through the FCT (Portuguese Foundation for Science and Technology) within project «Reminds»/ UTAP-ICDT/EEI-CTP/0022/2014.

\section{References}

1. Swan, K., Shen, J. and Hiltz, S.R.: Assessment and Collaboration in Online Learning. In J. Asynchronous Learning Networks, vol. 10, no. 1, pp. 45-61. (2006)

2. Burnett, B. and Roberts, A.: Online Collaborative Assessment: Unpacking Process and Product. In Assessing Online Learning, P. Comeaux. (eds.) pp. 55-71. Jossey-Bass (2005)

3. Hoffman, J. and Rogelberg, S.: All Together Now? College Students' Preferred Project Group Grading Procedures. In Group Dynamics: Theory, Research, and Pratice, vol. 5, no. 1, pp. 33-40. (2001)

4. Chyng, Y.J., Steinfeld, C. and Pfaff, B.: Supporting Awareness among Virtual Teams in a Web-Based Collaborative System: The TeamSCOPE System. In ACM SIGGROUP Bulletin, vol. 21, no. 3, pp. 28-34. (2000)

5. Boud, D., Cohen, R. and Sampson, A.J.: Peer Learning and Assessment. In Assessment and Evaluation in Higher Education, vol. 24, no. 4, pp. 413-426. (1999).

6. Strijos, J.W.: Assessment of (Computer-Supported) Collaborative Learning. In IEEE Transactions on Learning Technologies, Vol. 4, no. 1, pp. 59-70. (2011)

7. Gilies, R. M.: Cooperative Learning: Integrating Theory and Practice. Sage (2007)

8. Lee, E., Chan, C. and Aalst, J.: Students Assessing Their Own Collaborative Knowledge Building. In International Journal of Computer-Supported Collaborative Learning, vol. 1, no. 1, pp. 57-87, (2006)

9. Schummer, T., Strijos, J. and Berkel, T.: Measuring Group Interaction during CSCL. In Proceedings of Computer Supported Collaborative Learning: Learning (CSCL ' 05), pp. 567-576, (2005)

10. Fidalgo-Blanco, Á., Sein-Echaluce, M. L., García-Peñalvo, F. J., and Conde, M. Á.: Using Learning Analytics to improve teamwork assessment. Computers in Human Behavior, 47, pp. 149-156, (2015)

11. Figueira, A.: Orchestrating Online Group Work While Assessing Individual Participations. In Proceedings of INTED2015, pp.2996-3005. Madrid (2015) 